\section{Mortalidade infantil e evitabilidade em Mato Grosso do Sul, Brasil, 2000 a 2002}

\author{
Infant mortality and its preventability in Mato \\ Grosso do Sul State, Brazil, 2000-2002
}

\footnotetext{
1 Centro de Ciências Biológicas e da Saúde, Universidade Federal de Mato Grosso do Sul, Campo Grande, Brasil.

2 Universidade Católica Dom Bosco, Campo Grande, Brasil. 3 Faculdade de Medicina, Universidade Federal de Mato Grosso do Sul, Campo Grande, Brasil.

Correspondência A. L. G. S. Gastaud Departamento de Tecnologia de Alimentos e Saúde Pública, Centro de Ciências Biológicas e da Saúde, Universidade Federal de Mato Grosso do Sul. Rua Airton Rodrigues Leite 67, Campo Grande, MS 79022-066, Brasil. anagastaud@hotmail.com
}

\begin{abstract}
This retrospective study describes the epidemiological profile of infant mortality in 2000-2002 in 16 counties in Mato Grosso do Sul State, Brazil, and evaluates the preventability of such deaths, using the International Statistical Classification of Diseases and Related Health Problems (ICD10) and the List of Causes of Death Preventable by Interventions in the Setting of the Brazilian Unified National Health System in Children Under Five Years. Perinatal causes accounted for $54.3 \%$ of the 1,537 deaths, according to the Mortality Information System; congenital anomalies 14.9\%; infectious and parasitic diseases 9.4\%; and respiratory disorders 7\%. A full $73.1 \%$ of deaths were preventable, and most (69.5\%) resulted from inadequate prenatal, obstetric, and neonatal care. Additional causes were related to vaccine prevention (0.7\%), diagnosis and treatment (10.4\%), and health promotion (11.2\%). Causes not entirely avoidable accounted for $24.3 \%$ of deaths. The findings emphasize the relevance of monitoring adverse maternal-infant conditions and events, particularly to reduce early neonatal mortality ( $\leq 7$ days of life).
\end{abstract}

Infant Mortality; Maternal and Child Health; Epidemiological Surveillance
Ana Lúcia Gomes da Silva Gastaud 1

Michael Robin Honer 2

Rivaldo Venancio da Cunha 3

\section{Introdução}

A área materno-infantil se constitui em importante parâmetro para avaliação das condições de vida e saúde de uma população e em indicador da adequação da assistência obstétrica e neonatal, bem como do impacto de programas de intervenção nessa área.

Indicadores de saúde são utilizados para descrever uma situação existente e avaliar mudanças ou tendências durante certo período. O coeficiente de mortalidade infantil (CMI), por exemplo, está relacionado com a morte de nascidos vivos no primeiro ano de vida; o coeficiente de mortalidade materna (CMM), por sua vez, relaciona-se a óbitos de mulheres até 42 dias após o parto.

Os dados que servem de base a tais indicadores provêm principalmente da área de vigilância epidemiológica, conceituada como "um conjunto de ações que proporciona o conhecimento, a detecção ou prevenção de qualquer mudança nos fatores determinantes e condicionantes da saúde individual ou coletiva, com a finalidade de recomendar e adotar as medidas de prevenção e controle das doenças ou agravos" 1 (Capítulo I, Art. $\left.6^{\mathrm{o}}, \S 2^{\circ}\right)$.

O desenvolvimento de programas no Sistema Único de Saúde (SUS), especialmente aqueles que buscam reduzir a mortalidade infantil, exige uma base de dados confiável e um fluxo de notificação que expresse quantitativa e qualitativa- 
mente a complexidade da inter-relação das condições sócio-econômicas, ambientais, culturais e genéticas de uma dada população.

Segundo a Secretaria de Estado de Saúde de Mato Grosso do Sul (SES-MS) 2, até o ano de 2000 as mortes infantis ocorreram principalmente em menores de sete dias e por afecções originadas no período perinatal. Verificam-se também, nessa faixa etária, óbitos por malformações congênitas, deformidades e anomalias cromossômicas. As mortes em crianças de 28 dias a menores de um ano ocorreram em percentuais inferiores e predominantemente por doenças infecciosas e parasitárias, doenças do aparelho respiratório, doenças endocrinometabólicas e desnutrição.

As causas encontradas revelaram a potencial evitabilidade desses eventos, uma vez que se constituem em "mortes preveníveis, total ou parcialmente, por ações efetivas dos serviços de saúde que estejam acessíveis em um determinado local e época" 3 (p. 233).

O conjunto de dados aqui apresentados constitui-se em resultado parcial de estudo retrospectivo realizado com base em projeto de pesquisa intitulado A Mortalidade Materna e Mortalidade Infantil em Mato Grosso do Sul de 2000 a 2002 4, validado em curso de doutorado em Ciências da Saúde, do Programa Multiinstitucional de Pós-Graduação em Ciências da Saúde, Convênio Rede Centro-Oeste, Universidade de Brasília (UnB)/Universidade Federal de Goiás (UFG)/Universidade Federal de Mato Grosso do Sul (UFMS).

Este artigo tem como objetivo caracterizar o perfil epidemiológico da mortalidade infantil em 16 municípios de Mato Grosso do Sul, Brasil, e avaliar seu grau de evitabilidade.

\section{Metodologia}

O presente estudo compreendeu 16 dos $78 \mathrm{mu}$ nicípios sul-mato-grossenses, cuja inclusão foi estabelecida por critério de corte do projeto de pesquisa citado (Tabela 1). Os municípios incluídos perfazem aproximadamente $60 \%$ da população do Estado. Mato Grosso do Sul abrange 18\% da extensão territorial da Região Centro-Oeste e tem por capital Campo Grande. Faz fronteira seca com dois países: Paraguai e Bolívia. Dos 16 municípios selecionados, nove contam somente com serviços de atenção básica em saúde na rede pública.

Utilizou-se como fonte de dados o Sistema de Informações sobre Mortalidade (SIM) da SES-MS 2, sendo as causas básicas de morte identificadas segundo a Classificação Internacional de Doenças, 10a revisão (CID-10) 5 .
Na caracterização do perfil epidemiológico, as causas das 1.537 mortes infantis foram agrupadas por idade (menores de 7 dias; de 7 a 27 dias; de 28 dias a menores de 1 ano). Foram identificados também o sexo e local de procedência dos nascidos vivos, assim como o local de parto.

Posteriormente, foi realizada avaliação da evitabilidade dos óbitos infantis, mediante a utilização da Lista de Causas de Morte Evitáveis por Intervenções no Âmbito do Sistema Único de Saúde do Brasil 3. Essa listagem, proposta por um grupo de especialistas constituído pelo Ministério da Saúde, foi elaborada a partir de uma revisão da literatura relativa à base conceitual e empírica do tema, publicada de 1975 a 2004, tendo por referência a tecnologia disponível no SUS $6,7,8,9,10,11,12,13$. Essa listagem abrange as seguintes categorias:

1. Causas evitáveis reduzíveis por:

1.1. ações de imunoprevenção;

1.2. adequada atenção à mulher na gestação e no parto, assim como ao recém-nascido;

1.2.1. adequada atenção à mulher na gestação;

1.2.2. adequada atenção à mulher no parto;

1.2.3. adequada atenção ao recémnascido;

1.3. ações adequadas de diagnóstico e tratamento;

1.4. ações adequadas de promoção à saúde, vinculadas a ações adequadas de atenção à saúde.

2. Causas mal definidas.

3. Demais causas (não claramente evitáveis).

Para cada um desses subgrupos, dispõe-se de uma relação de diagnósticos identificados pela CID-10 5 .

O protocolo de pesquisa foi aprovado pelo Comitê de Ética de Pesquisa com Seres Humanos da UFMS, de acordo com o protocolo $\mathrm{n}^{\circ}$. 183, de novembro de 2002.

\section{Resultados}

\section{Caracterização do perfil epidemiológico}

Constatou-se que 54,9\% (844) das mortes infantis ocorreram em crianças do sexo masculino e $38,9 \%$ (598), do sexo feminino. Em 6,2\% (95) não houve identificação de sexo nos registros.

Do total dessas mortes, 83,8\% (1.288) foram de crianças moradoras da zona urbana, $10,7 \%$ (164), de aldeias indígenas, e 4,9\% (75), da zona rural. Em 0,6\% (10) não havia indicação de procedência. A significativa maioria dos óbitos 
Mortes infantis em 16 municípios de Mato Grosso do Sul, Brasil, 2000 a 2002.

\begin{tabular}{|c|c|c|c|c|}
\hline Município & 2000 & 2001 & 2002 & Total \\
\hline Amambaí & 20 & 32 & 30 & 82 \\
\hline Aquidauana & 25 & 29 & 18 & 72 \\
\hline Bonito & 7 & 6 & 7 & 20 \\
\hline Brasilândia & 9 & 12 & 4 & 25 \\
\hline Campo Grande & 212 & 191 & 167 & 570 \\
\hline Chapadão do Sul & 3 & 6 & 2 & 11 \\
\hline Corumbá & 87 & 78 & 81 & 246 \\
\hline Dourados & 118 & 111 & 74 & 303 \\
\hline Eldorado & 8 & 3 & 4 & 15 \\
\hline Guia Lopes da Laguna & 1 & 3 & 5 & 9 \\
\hline Itaquiraí & 4 & 7 & 5 & 16 \\
\hline Paranhos & 12 & 17 & 14 & 43 \\
\hline Ribas do Rio Pardo & 8 & 10 & 9 & 27 \\
\hline Santa Rita do Pardo & 3 & 1 & 3 & 7 \\
\hline Terenos & 3 & 2 & 5 & 10 \\
\hline Três Lagoas & 25 & 36 & 20 & 81 \\
\hline Total & 545 & 544 & 448 & 1.537 \\
\hline
\end{tabular}

$(86,7 \% ; 1.332 / 1.537)$ ocorreu em hospitais, principalmente naqueles localizados na cidade de moradia da criança $(80,3 \%)$.

Considerando-se o total de óbitos, identificou-se (Tabela 2) maior número de mortes infantis $(54,3 \%$; $834 / 1.537)$ por afecções originadas no período perinatal, sendo essas afecções, na maioria, em menores de sete dias $(83,4 \%$; 668/801). Tal predominância ocorreu em Bonito, Brasilândia, Campo Grande, Chapadão do Sul, Corumbá, Dourados, Itaquiraí, Terenos e Três Lagoas, ou seja, em 9 dos 16 municípios estudados.

As malformações congênitas, que constituíram o segundo grupo preponderante de causas (14,9\%; 230/1.537), foram responsáveis por números aproximadamente iguais de mortes em todas as faixas etárias. Em menor percentual e com perfil assemelhado, ocorreram mortes por doenças infecciosas e parasitárias $(9,4 \% ; 145 / 1.537)$ e do aparelho respiratório $(8,7 \% ; 134 / 1.537)$, as quais predominaram na faixa etária de 28 dias a menores de um ano.

Nas afecções originadas no período perinatal, identificaram-se principalmente causas e/ ou transtornos maternos por complicações da gravidez, do trabalho de parto e do parto (P00P04); pela duração da gestação e crescimento fetal (P05-P08); por agravos respiratórios e cardiovasculares específicos do período perinatal (P20-P29); por infecções específicas do período perinatal (P35-P39) e por agravos endócrinos e metabólicos transitórios específicos do feto e do recém-nascido (P70-P78).

Considerando-se o total de mortes infantis no período (1.537), as doenças denominadas como "outras afecções respiratórias originadas no período perinatal” (P28; 10,7\%) foram as causadoras do maior número de eventos, seguidas de "desconforto respiratório do recém-nascido" (P22; 9,4\%), "septicemia bacteriana do recémnascido” (P36; 6,3\%), "pneumonias” (J18; 6,1\%), "diarréias e gastroenterites de origem infecciosa" (A09; 5,6\%), "malformações congênitas do coração" (Q24; 4,9\%) e "transtornos relacionados com gestação de curta duração e peso baixo ao nascer" (P07; 4,6\%). Observe-se que o somatório dessas causas representou $42,7 \%$ da totalidade das mortes.

Destaca-se que as malformações congênitas do coração, dentro do grupo das anomalias congênitas, constituíram 37,4\% (86/230) das mortes nesse grupo.

As mortes diagnosticadas por sintomas, sinais e achados anormais de exames clínicos de laboratório e por causas externas perfizeram aproximadamente $6 \%$.

Como mostra a Tabela 3, e segundo dados do Ministério da Saúde 14, a maioria dos municípios (Amambaí, Aquidauana, Bonito, Brasilândia, Chapadão do Sul, Corumbá, Dourados, Eldorado, Guia Lopes da Laguna, Itaquiraí, Paranhos, Ribas do Rio Pardo, Santa Rita do Pardo) e também 
Tabela 2

Causas de mortalidade infantil, por faixas etárias, em 16 municípios de Mato Grosso do Sul, Brasil, 2000 a 2002.

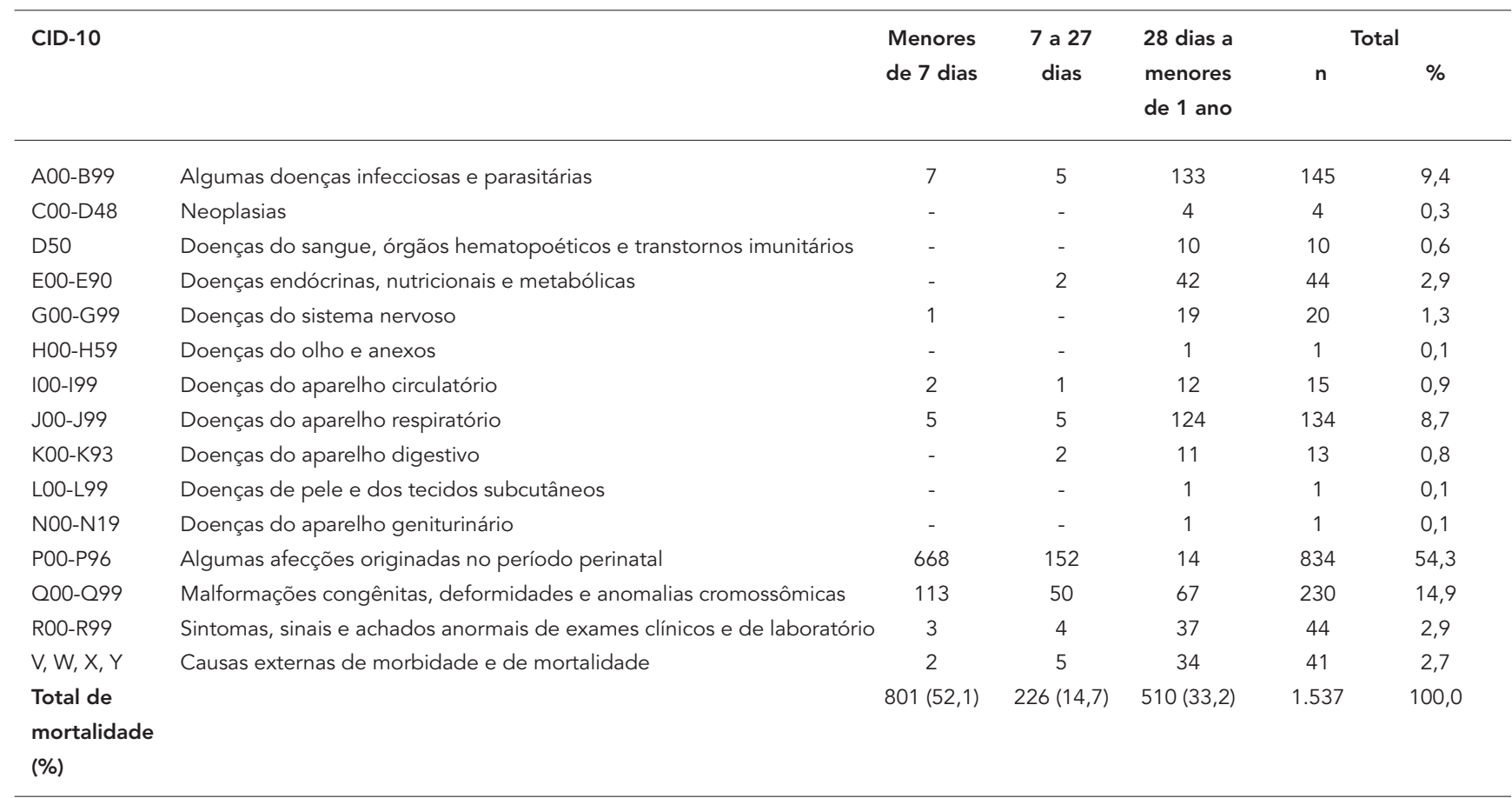

CID-10: Classificação Internacional de Doenças, 10ạ revisão 5 .

Tabela 3

Coeficiente de mortalidade infantil (CMI) em 16 municípios de Mato Grosso do Sul, Brasil, 2000 a 2002.

\begin{tabular}{|c|c|c|c|}
\hline \multirow[t]{2}{*}{ Município } & \multicolumn{3}{|c|}{ CMI } \\
\hline & 2000 & 2001 & 2002 \\
\hline Amambaí & 30,1 & 47,6 & 41,4 \\
\hline Aquidauana & 24,9 & 30,4 & 19,2 \\
\hline Bonito & 20,1 & 16,8 & 19,4 \\
\hline Brasilândia & 42,3 & 52,9 & 19,6 \\
\hline Campo Grande & 16,1 & 15,6 & 13,5 \\
\hline Chapadão do Sul & 20,4 & 37,7 & 10,6 \\
\hline Corumbá & 36,6 & 31,6 & 32,3 \\
\hline Dourados & 32,8 & 30,7 & 22,6 \\
\hline Eldorado & 28,3 & 9,5 & 12,5 \\
\hline Guia Lopes da Laguna & 4,9 & 14,0 & 26,5 \\
\hline Itaquiraí & 15,4 & 25,6 & 15,7 \\
\hline Paranhos & 51,7 & 76,9 & 54,5 \\
\hline Ribas do Rio Pardo & 27,9 & 34,6 & 29,2 \\
\hline Santa Rita do Pardo & 42,3 & 10,9 & 31,3 \\
\hline Terenos & 19,4 & 12,1 & 30,1 \\
\hline Três Lagoas & 18,1 & 26,0 & 13,9 \\
\hline Mato Grosso do Sul & 23,8 & 23,7 & 20,1 \\
\hline
\end{tabular}


o Estado de Mato Grosso do Sul apresentaram CMIs classificados como taxas médias de mortalidade infantil (20 a 49 mortes por mil nascidos vivos). Campo Grande, Três Lagoas e Guia Lopes da Laguna apresentaram taxas baixas (menos de 20 mil mortes por nascidos vivos). Em Terenos, o CMI em 2002 elevou-se, alcançando a taxa média. Os municípios apresentaram, em sua quase totalidade, CMI maior que o estadual.

Em Amambaí e Paranhos, o elevado CMI deveu-se principalmente a óbitos de crianças indígenas. Juntamente com Dourados, estes são os municípios sul-mato-grossenses com maior população indígena (Tabela 4). Nesses três municípios, as mortes indígenas constituíram, em relação à totalidade de óbitos, os seguintes percentuais: 60,9\% em Amambaí; 39,5\% em Paranhos; $27,4 \%$ em Dourados. Cerca de $40 \%$ do total desses óbitos, nos três municípios, ocorreram em crianças não hospitalizadas.

O somatório das mortes infantis indígenas, como mostra a Tabela 4, também revela predominância de causas perinatais. Contudo, pelo fato de em Amambaí e Paranhos as mortes ocorrerem em maior percentual na faixa etária de 28 dias a menores de 1 ano, tem-se uma elevação do número de eventos causados por doenças infecciosas e parasitárias, assim como respiratórias, conforme mostra a comparação entre as Tabelas 2 e 4.

\section{Causas de mortalidade infantil quanto ao grau de evitabilidade}

Dos óbitos ocorridos nos 16 municípios (Tabela 5), 73,1\% (1.124/1.537) constituíram mortes infantis evitáveis, das quais $69,5 \%(781 / 1.124)$ foram consideradas reduzíveis por adequada atenção à mulher na gestação e parto e, predominantemente $(69,7 \%$; $544 / 781)$, ao recémnascido.

Como anteriormente apontado, destacaramse os óbitos que envolveram transtornos relacionados com gestação de curta duração e peso baixo ao nascer (P07), hipóxia intra-uterina e asfixia ao nascer (P20-P21), transtornos respiratórios e cardiovasculares específicos do período perinatal (P23, P25-P28) e desconforto respiratório do recém-nascido (P22). Esses eventos, em seu conjunto, representaram 54,4\% (612/1.124) do item citado, assim como cerca de $40 \%$ de todas as mortes.

Ocorreram também, em percentuais assemelhados, óbitos reduzíveis por ações adequadas de diagnóstico e tratamento $(10,4 \% ; 160 / 1.537)$ e por ações de promoção à saúde, vinculadas a ações adequadas de atenção à saúde (11,2\%; 173/1.537), com predomínio de mortes por pneumonia (J12-J18), doenças infecciosas intestinais
(A00-A09) e deficiências nutricionais (E40-E64), as quais somaram $14,4 \%(222 / 1.537)$.

As causas mal definidas representaram $2,6 \%$ (40/1.537). Destaque-se o fato de $24,3 \%$ (373/1.537) dos eventos não serem claramente identificados como evitáveis, ocorrendo predominância $(60,6 \%$; 226/373) de malformações congênitas. Enquadram-se neste item, e em maior percentual $(39,9 \%$; 149/373), os transtornos cardíacos inseridos no grupo de doenças do aparelho circulatório (I27, I31, I33, I38, I42, I50, I51, I60, I61, J39), as afecções originadas no perinatal (P29) e as malformações congênitas (Q20-Q25).

\section{Discussão}

Os resultados apontam a necessidade de ampla avaliação que integre todos os procedimentos incluídos na área materno-infantil.

O maior número de mortes se mantém em crianças menores de sete dias, observado igualmente, em outros estudos, em período assemelhado 8,15,16. O predomínio de mortes infantis encontrado em crianças do sexo masculino é uma tendência observada na literatura, em dados do período de 1980 a 2003, nas idades de 0 a 16 anos 17,18 .

Os achados do presente estudo coincidem com diagnósticos relacionados nas listas especiais de tabulação para mortalidade infantil e da criança, integrantes do documento da CID-10 5, assim como estudos no Brasil que identificaram mortes infantis por afecções originadas no período perinatal, doenças infecciosas e parasitárias, doenças do aparelho respiratório e anomalias congênitas, de modo geral nesta ordem de importância. Os três primeiros motivos ocorreram, em sua maioria, em maior percentual nas camadas de mais baixa renda 19,20,21. Observe-se ainda que houve semelhança entre o somatório de causas de mortes predominantes identificadas na CID-10 e os da lista de causas de morte evitáveis utilizada.

Verificou-se que um terço das mortes evitáveis $(237 / 781)$ estavam relacionadas com adequada atenção à mulher na gestação e no parto. Em outros estados do Brasil, foram igualmente encontrados problemas graves na atenção à mulher no período gravídico-puerperal, influenciando o aumento do CMI, aumento esse reforçado pelas desigualdades sociais 22,23,24,25.

Victora et al. 26, Costa et al. 27 e Barros et al. 28 constataram, em Município do Rio Grande do Sul, Brasil, a influência na saúde infantil de uma série de fatores perinatais, demográficos, ambientais, alimentares e assistenciais. Identificaram, como fatores de risco significativos para mortalidade 
Tabela 4

Causas de mortalidade infantil indígena nos Municípios de Amambaí, Dourados e Paranhos, Mato Grosso do Sul, Brasil, 2000 a 2002.



CID-10: Classificação Internacional de Doenças, 10ạ revisão 5 .

Classificação de mortes infantis segundo critérios de evitabilidade, em 16 municípios de Mato Grosso do Sul, Brasil, 2000 a 2002.

\begin{tabular}{|c|c|c|}
\hline Mortes & $\mathbf{n}$ & $\%$ \\
\hline \multicolumn{3}{|l|}{ 1. Causas evitáveis reduzíveis por: } \\
\hline 1.1. imunoprevenção & 10 & 0,7 \\
\hline \multicolumn{3}{|l|}{ 1.2. adequada atenção à mulher na gestação e parto e ao recém-nascido } \\
\hline 1.2.1. adequada atenção à mulher na gestação & 116 & \\
\hline 1.2.2. adequada atenção à mulher no parto & 121 & \\
\hline 1.2.3. adequada atenção ao recém-nascido & 544 & \\
\hline Subtotal 1.2.1. + 1.2.2. + 1.2.3. & 781 & 50,8 \\
\hline 1.3. ações adequadas de diagnóstico e tratamento & 160 & 10,4 \\
\hline 1.4. ações adequadas de promoção à saúde, vinculadas a ações adequadas de atenção à saúde & 173 & 11,2 \\
\hline Subtotal 1.1. + 1.2. + 1.3. + 1.4. & 1.124 & 73,1 \\
\hline 2. Causas mal definidas & 40 & 2,6 \\
\hline 3. Demais causas (não claramente evitáveis) & 373 & 24,3 \\
\hline Total 1. + 2. + 3. & 1.537 & 100,0 \\
\hline
\end{tabular}


infantil, as condições sócio-econômicas familiares, assim como baixo peso do recém-nascido, associação com escolaridade da gestante, fumo na gravidez e qualidade do pré-natal oferecido. Os dois estudos também revelaram índices de mortes por imaturidade pulmonar e asfixia cerca de três vezes maiores que os das demais causas, evidenciando deficiências no pré-natal e falhas na assistência ao parto.

Identificou-se que nem sempre a causa individual, com maior percentual, de óbito infantil em um município enquadrou-se no grupo da CID-10 em que ocorreu o maior número de mortes. Em Amambaí, por exemplo, observou-se predominância de mortes no grupo de causas perinatais - embora um maior número de crianças tenha morrido por pneumonia, o que se insere no grupo de causas respiratórias - e em crianças de 28 dias a menores de 1 ano. Dessa forma, evidenciase que a avaliação da mortalidade infantil não pode basear-se exclusivamente no somatório de causas nos diferentes grupos de diagnóstico.

Essa diferença de resultados, como já exposto, deve-se a mortes de crianças indígenas. A mortalidade infantil indígena apresentou maior percentual de causas relacionadas a doenças infecciosas e parasitárias e desnutrição, com predomínio da faixa etária de 28 dias a menores de 1 ano, agravando-se essa realidade pelo percentual significativo de óbitos ocorridos fora de hospitais.

Esses dados se assemelham aos de Ribas et al. ${ }^{29}$, que descreveram as condições de saúde e nutrição de crianças indígenas Teréna, residentes em aldeias de Mato Grosso do Sul. Em tal estudo, foi constatada desnutrição infantil elevada, associada às precárias condições sócio-econômicas, sendo também verificada baixa cobertura de prénatal, agravada pelo número de partos domiciliares, com incidência de baixo peso ao nascer.

Não se pode deixar de considerar a importância do peso da criança ao nascer, visto que esse fator tem-se mostrado de risco. Entretanto, o grupo de especialistas que elaborou a lista de mortes evitáveis em menores de cinco anos ${ }^{3}$ não optou pela definição de pontos de corte segundo o peso dos recém-nascidos, para posterior consideração da evitabilidade do óbito infantil.

O número elevado de óbitos por causas respiratórias em recém-nascidos, a prevalência de causas enquadradas como evitáveis e a constatação de que a maioria dos partos foi realizada em hospitais reforçam a importância de como os serviços se estruturam para lidar com situações de risco, no parto e puerpério imediato. Essa questão relaciona-se ainda com o fato de que nove municípios contam somente com serviços de atenção básica.
O comparativo de resultados deste estudo com o perfil epidemiológico dos 78 municípios de Mato Grosso de Sul identificou percentuais assemelhados de óbitos infantis nos mesmos grupos de causas e na mesma ordem de importância 2,30. Tal semelhança ocorre também com o conjunto de dados da capital do estado.

A comparação da mortalidade infantil no período estudado com dados obtidos de 2003, 2004 e 2005 evidenciou, igualmente, não haver alteração importante no perfil de mortalidade infantil no estado 2. Persistem as causas principais em cada grupo identificadas pela CID-10 5, sendo essa realidade próxima à dos demais estados do Brasil 31 .

A constatação de óbitos em maior número por causas evitáveis e na faixa etária de 28 dias a 1 ano em municípios de menor poder aquisitivo e com a existência de grupos populacionais com inúmeras dificuldades para subsistência permite inferir que as características sócio-econômicas de uma população poderão, negativa ou positivamente, ser potencializadoras da qualidade de vida e saúde. Por essa razão, Granda \& Breilh 32 , assim como outros pesquisadores $21,22,27,28$, entendem que o fato epidemiológico e o processo saúde-doença são conseqüências de um complexo processo de diferentes dimensões, consideradas como fatores de risco.

Reforça-se o entendimento de que a redução da morbimortalidade infantil e o monitoramento das condições de saúde, principalmente materno-infantil, não podem se ater a um único indicador ou fato, já que, no perfil epidemiológico de Mato Grosso do Sul, persistem situações de risco e, principalmente, porque se verifica que cerca de $80 \%$ dessas mortes caracterizam-se como evitáveis ou reduzíveis.

Evidencia-se, como afirma Lansky et al. 9, que a magnitude e a estrutura de causalidade da mortalidade infantil constituem-se como "evento-sentinela" da qualidade da assistência à saúde de uma população.

Dessa forma, os Comitês de Mortalidade Materna e os de Prevenção do Óbito Infantil e Fetal 14,33, cuja implantação é proposta pelo Ministério da Saúde, por suas características e objetivos, podem ser instâncias contributivas para monitoramento das condições de saúde da população e da forma como se dá o atendimento em face das diferentes demandas.

Impõem-se ações intersetoriais, tais como expansão do saneamento básico, aumento da disponibilidade de alimentos aos grupos populacionais de risco para desnutrição e ações educativas. Torna-se igualmente necessário submeter o crescimento da rede básica de saúde e da rede hospitalar a critérios geográficos e populacio- 
nais, de modo a facilitar o acesso da população a essas redes.

Por fim, acredita-se, como Ribas et al. ${ }^{29}$, que a interação entre estudos de cunho acadêmico e aqueles demandados no cotidiano dos serviços públicos de saúde possam contribuir com as transformações necessárias para a melhoria dos indicadores materno-infantis, bem como para o desenvolvimento das políticas públicas de saúde, de suas estruturas organizacionais e da qualificação de seus recursos humanos.

\section{Conclusão}

A avaliação do conjunto de causas comprova a prevalência de mortes infantis por causas evitáveis e sua possível relação com a situação sócio-econômica familiar, como se constatou nas mortes infantis indígenas. Pode se dizer que indicadores como o CMI apresentou valores variáveis inversamente proporcionais ao grau de desenvolvimento da região.

Em se tratando mortalidade infantil, os dados indicam a possibilidade de se identificarem os grupos de risco, visto que se evidenciaram avanços na organização e funcionamento do sistema de informações sobre saúde, principalmente em nível estadual. Contudo, avalia-se que o monitoramento da realidade materno-infantil exige o pleno funcionamento de instâncias estruturadas para tal fim, como é o caso dos Comitês de Mortalidade Materna e dos de Prevenção do Óbito Infantil e Fetal.

Verifica-se que muitas causas identificadas poderiam ser evitadas com adoção de medidas de prevenção e promoção, tais como programas de imunização, pré-natal, puericultura e planejamento familiar, além de ações para qualificação das estruturas de saúde e de recursos humanos, principalmente nas unidades básicas de saúde e na rede hospitalar.

Recomenda-se o enfoque de evitabilidade para a abordagem da mortalidade infantil, que mostrou ser indicador de efetividade dos serviços de saúde.

Deve se destacar que as iniciativas, por parte dos órgãos municipais e estadual de saúde, devem contemplar uma avaliação não apenas de aspectos curativos e quantitativos, mas preventivos e qualitativos relacionados com o processo saúde-doença e com a qualidade de vida.

A redução dos indicadores de mortalidade infantil demanda, assim, ações de caráter político-institucional, incluindo, além da detecção precoce de problemas existentes antes ou durante a gravidez, o término da dissociação entre a assistência pré-natal e a do parto e puerpério, enfocando o princípio da integralidade da assistência à mulher e à criança.

\section{Resumo}

Este estudo retrospectivo caracteriza o perfil epidemiológico da mortalidade infantil em 16 municípios sulmato-grossenses em 2000-2002 e avalia seu grau de evitabilidade, utilizando a Classificação Internacional de Doenças, 10a Revisão (CID-10) e a Lista de Causas de Morte Evitáveis por Intervenções no Âmbito do Sistema Único de Saúde do Brasil. Dados do Sistema de Informações sobre Mortalidade (SIM) revelaram óbitos por causas perinatais em 54,3\% dos 1.537 casos; anomalias congênitas em 14,9\%; doenças infecciosas e parasitárias em $9,4 \%$ e doenças do aparelho respiratório em 7\%. As mortes evitáveis e reduzíveis perfi- zeram 73,1\%, na maior parte (69,5\%) decorrendo de inadequada atenção à mulher na gestação e parto e ao recém-nascido. Outras causas relacionaram-se à imunoprevenção (0,7\%), ações de diagnóstico e tratamento $(10,4 \%)$ e promoção à saúde $(11,2 \%)$. As mortes por causas não claramente evitáveis totalizaram $24,3 \%$. Os resultados reforçam a relevância da monitorização de agravos materno-infantis para redução da mortalidade infantil, principalmente em menores de sete dias.

Mortalidade Infantil; Saúde Materno-Infantil; Vigilância Epidemiológica 


\section{Colaboradores}

A. L. G. S. Gastaud foi a principal responsável pela realização da pesquisa e redação do artigo. M. R. Honer foi responsável pela orientação da pesquisa e revisão do trabalho. R. V. Cunha participou da análise dos resultados obtidos e da revisão crítica final do trabalho.

\section{Referências}

1. Brasil. Lei Federal no. 8.080, de 19 de setembro de 1990. Dispõe sobre promoção, proteção, e recuperação da saúde, a organização e funcionamento dos serviços. Diário Oficial da União 1990; 20 set.

2. Coordenadoria de Informação e Desempenho da Saúde, Secretaria de Estado de Saúde do Mato Grosso do Sul. Estatísticas de saúde: mortalidade e nascidos vivos. v. 1. Campo Grande: Secretaria de Estado de Saúde do Mato Grosso do Sul; 2003.

3. Malta DC, Duarte EC, Almeida MF, Dias MAS, Morais Neto OL, Moura L, et al. Lista de causas de mortes evitáveis por intervenções do Sistema Único de Saúde do Brasil. Epidemiol Serv Saúde 2007; 16:233-44.

4. Gastaud ALGS. A mortalidade materna e mortalidade infantil em Mato Grosso do Sul, Brasil, de 2000 a 2002 [Tese de Doutorado]. Brasília: Universidade de Brasília; 2007.

5. Organização Mundial da Saúde. Classificação estatística internacional de doenças e problemas relacionados à saúde, 10ạ revisão. 8a Ed. São Paulo: EdUSP; 2000.

6. Hartz ZMA, Champagne F, Leal MC, Contandriopoulos AP. Mortalidade infantil "evitável" em duas cidades do Nordeste do Brasil: indicador de qualidade do sistema local de saúde. Rev Saúde Pública 1996; 30:310-8.

7. Vidal AS, Frias PG, Barreto FMP, Vanderlei LCM, Felisberto E. Óbitos infantis evitáveis em hospital de referência estadual do Nordeste brasileiro. Rev Bras Saúde Matern Infant 2003; 3:281-9.

8. Ferrari LSL, Brito ASJ, Carvalho ABR, Gonzáles MRC. Mortalidade neonatal no Município de Londrina, Paraná, Brasil, nos anos de 1994, 1999 e 2002. Cad Saúde Pública 2006; 22:1063-71.

9. Lansky S, França E, Leal MC. Mortalidade perinatal e evitabilidade: revisão da literatura. Rev Saúde Pública 2002; 36:759-72.

10. Fundação Sistema Estadual de Análise de Dados. Agrupamento das causas de morte dos menores de um ano segundo critério de evitabilidade das doenças. São Paulo: Fundação Sistema Estadual de Análise de Dados; 1988.

11. Ortiz-Flores LP. Características da mortalidade neonatal no estado de São Paulo [Tese de Doutorado]. São Paulo: Universidade de São Paulo; 1999.
12. Ministério da Saúde. Portaria $\mathrm{n}^{\circ}$. 723 , de 10 de maio de 2001. Pacto de indicadores da atenção básica para o ano de 2001. Diário Oficial da União 2001; 11 mai.

13. Wigglesworth JS. Monitoring perinatal mortality: a pathophysiological approach. Lancet 1980; 2: 684-6.

14. Ministério da Saúde. Manual dos Comitês de Prevenção do Óbito Infantil e Fetal. Brasília: Ministério da Saúde; 2005.

15. Castro ECM, Leite AJM. Mortalidade hospitalar dos recém-nascidos com peso de nascimento menor ou igual a $1.500 \mathrm{~g}$ no Município de Fortaleza. J Pediatr 2007; 83:27-32.

16 Gomes MASM, Lopes JMA, Moreira MEL, Gianini NOM. Assistência e mortalidade neonatal no setor público do Município do Rio de Janeiro, Brasil: uma análise do período 1994/2000. Cad Saúde Pública 2005; 21:1269-77.

17. U. S. Department of Health and Human Services/ National Center for Health Statistics, Centers for Disease Control and Prevention. Health, United States, 2005: with chartbook on trends in the heath of Americans. Washington DC: U. S. Department of Health and Human Services; 2005.

18. Schmitz BAS, Bezerra VLV, Turnes O. Mortalidade infantil no Distrito Federal segundo sexo e causa básica de morte: 1988-1989. Rev Saúde Dist Fed 2000; 11:14-21.

19. Lansky S, França E, Leal MC. Mortes perinatais evitáveis em Belo Horizonte, Minas Gerais, Brasil, 1999. Cad Saúde Pública 2002; 18:1389-400.

20. Andrade CLT, Szwarcwald CL, Gama SGN, Leal MC. Desigualdades sócio-econômicas do baixo peso ao nascer e da mortalidade perinatal do Município do Rio de Janeiro, 2001. Cad Saúde Pública 2004; 20 Suppl 1:S44-51.

21. Costa MCN, Azi PA, Paim JS, Silva LMV. Mortalidade infantil e condições de vida: a reprodução das desigualdades sociais em saúde na década de 90. Cad Saúde Pública. 2001; 17:555-67.

22. Vieira LAS. Mortalidade infantil: desigualdades sociais e em saúde [Dissertação de Mestrado]. Florianópolis: Universidade Federal de Santa Catarina; 2000. 
23. Lansky S, França E, César CC, Monteiro Neto LC, Leal MC. Mortes perinatais e avaliação da assistência ao parto em maternidades do Sistema Único de Saúde, em Belo Horizonte, Minas Gerais, Brasil, 1999. Cad Saúde Pública 2006; 22:117-30.

24. Avanços na luta contra a mortalidade materna e neonatal. JAMB 2006; 47:20-1.

25. Laurenti R, Jorge MHPM, Gotlieb SLD. A mortalidade materna nas capitais brasileiras: algumas características e estimativa de um fator de ajuste. Rev Bras Epidemiol 2004; 7:458-9.

26. Victora CG, Barros FC, Tomasi E, Menezes AM, Horta BL, Weiderpass E, et al. Tendências e diferenciais na saúde materno-infantil: delineamento e metodologia das coortes de 1982 e 1993 de mães e crianças de Pelotas, Rio Grande do Sul. Cad Saúde Pública 1996; 12 Suppl 1:S7-14.

27. Costa JSD, Victora CG, Barros FC, Halpern R, Horta BL, Manzolli P. Assistência médica materno-infantil em duas coortes de base populacional no Sul do Brasil: tendências e diferenciais. Cad Saúde Pública 1996; 12 Suppl 1:S59-66.
28. Barros AJD, Santos IS, Victora CG, Albernaz EP, Domingues MR, Timm IK, et al. Coorte de nascimentos de Pelotas, 2004: metodologia e descrição. Rev Saúde Pública 2006; 40:408-12.

29. Ribas DLB, Sganzerla A, Zorzatto JR, Philippi ST. Nutrição e saúde infantil em uma comunidade indígena Terena, Mato Grosso do Sul, Brasil. Cad Saúde Pública 2001; 17:323-31.

30. Coordenadoria de Informação e Desempenho da Saúde, Secretaria de Estado de Saúde de Mato Grosso do Sul. Sistema de Informações sobre Mortalidade. http://www.saude.ms.gov.br (30/Jun/ 2006).

31. Ministério da Saúde/Organização Pan-Americana da Saúde. Painel de indicadores do SUS. Brasília: Ministério da Saúde; 2006.

32. Granda E, Breilh J. Saúde na sociedade. São Paulo: Editora Hucitec; 1993.

33. Ministério da Saúde. Manual de Comitês de Mortalidade Materna. 2a Ed. Brasília: Ministério da Saúde; 2002.

Recebido em 20/Jun/2007

Versão final reapresentada em 04/Dez/2007

Aprovado em 21/Dez/2007 